\title{
Research on the Application of Steel Plate Surface Defect Detection System Based on Machine Vision
}

\author{
Xianfeng Liu \\ Information Engineering College, Changzhou Vocational Institute of Mechatronic Technology, \\ Changzhou Jiangsu, 213164, China
}

Keywords: Machine vision technology,Steel plate surface defect, Camera lens, Calibration technology, Scratch length detection.

\begin{abstract}
The quality control and detection method for traditional artificial steel plate surface is quite time-consuming and with low detection efficiency, which cannot meet actual demand of modern industry. This paper bases on machine vision technology to study steel plate surface defect detection system, which relies on computer science, robots science and visual calibration technology, perfectly replaced artificial automatic detection technology, can realize automatic detection for steel plate surface defects, not only greatly improved detection efficiency and quality, but also decreased labor cost. This paper mainly studies and summaries three aspects of the election of visual detection camera and lens, analysis on machine visual calibration technology and steel plate surface defect detection from machine vision.
\end{abstract}

\section{Introduction}

Mechanical vision detection technology is based on machine vision technology research, it is professional detection technology, it basically can achieve complex technical contents of pattern recognition, vision understanding, also can realize important detection means like quantitative determination and noncontact measurement. Compared with traditional artificial detection technology, machine vision technology detection system more emphasizes on instantaneity and high-speed on the aspect of picture processing, in some industry detections, it can reach detection requirement that human eyes cannot reach (exceed the basic standard that human eye detection reaches $80 \%$ ), reaches $100 \%$ precise detection. And it is realized on the automatic and intelligent functions.

\section{Research on the election of camera and lens in the mechanical vision detection technology}

Mechanical vision detection technology is an interdiscipline involves artificial intelligence, computer science, picture processing, pattern recognition, its most important character is with fast speed, large amounts of information and multiple functions. Collect relevant valid data from detected pictures, transform the collected image data signals, coordinate detection result to output its main detection technology procedure as following:

Image collection of detected targets $\rightarrow$ transform image signal $\rightarrow$ detection result output

In the whole detection technology system, image collection is the initial foundation link, it determines the quality of the collected images, high-quality image results can simplify the follow-up image processing, to make detection precise reach a certain height, further to improve detection efficiency. Here, digital image processing[1] is the process to transform image signals into digital signals and use computer for processing, has following advantages: (1) good reproducibility; (2) high processing precision; (3) wide application; (4) good flexibility; (5) huge potential for information compression. Collection of targeted images should depend on camera and lens, it is crucial for the design and development of detection technology to study how to choose camera and lens. 


\section{Research on camera classification}

Camera is the key equipment for image collection of visual detection technology, the importance of its function is self-evident. There are many different kinds of cameras currently, in the actual detection process, we have to elect suitable camera to match the visual detection technology according to the real situation. From the camera classification standards, its key research part includes image sensor chip types, scanning mode, sensor structure features, resolution ratio, frequency response range and so on.

\section{Research on the election of image sensor technology}

Compared with traditional CCD, CMOS image sensor technology has many advantages and is more mature, it can array and simulate sensing units to integrate many digital functions on one chip to form complete picture pick-up system of visual detection technology. Application of this technology has great help on the simplification of system complexity, also can effectively reduce system construction cost and detection implementation cost.

From a technical perspective, CMOS image sensor has inherent column parallel readout mode, it can randomly read with high speed according to the pixel data, ant its power dissipation is just 1/10 of ordinary CCD image sensor.

\section{Election of camera}

According to the requirement of detection environment, if we carry out research on the steel plate surface defect detection, the detection precision of machine vision technology must reach $0.1 \mathrm{~mm}$ or ever over this. If the surface size of steel plate is within $220 \mathrm{~mm} * 50 \mathrm{~mm}$, its camera resolution ratio must reach $2200 * 500$ pixels, and better use multiple resolution power to ensure total pixels reach 12.4 million thus can meet detection demand. Generally speaking, the camera resolution ratio can be adjusted within 2256*1504 pixels, thus can maximum meet the detection demand of steel plate surface defect, and can maximum improve the treatment speed of detection images.

\section{Election of lens}

Len is also an important part affecting imaging quality, famous lens brand includes Schneider, Lycra, Zeiss.Camera lens is the key of the machine vision detection system, it is equivalent to the human crystalline lens, which can output sharp image for camera.During the actual image detection process, lens quality directly affect total mechanism of the system, therefore, lens election should be careful, the main considering objects include focal length, imaging plane, angle of view, working distance, depth of field, view and other important parameters. Above elements mainly satisfy the following relations:

$$
\begin{aligned}
& F_{1}=v \times D / V \\
& F_{2}=h \times D / H
\end{aligned}
$$

In above relations, $F_{1} 、 F_{2}$ refers focal length of lens both on the horizontal and vertical direction, while the actual focal length of lens is determined by the smaller one within $F_{1} 、 F_{2}, D$ refers maximum working distance, $v \times h$ refers the size of imaging plane. From the formula we also can know that when the view and the focal length of camera lens are negatively related, they must positively related with minimum working distance. That is to say, the greater the focal length of lens is, the smaller its view and horizon is, the farer the working distance is.

In the real detection process of steel plate surface defect, we must consider the real detection working distance, namely the real distance between steel plate and camera. Assume the detection working distance is $50 \mathrm{~cm}$, thus the lens focal length calculated from above formula should be around $15 \mathrm{~mm}$, thus camera lens should be $15 \sim 50 \mathrm{~mm}$ convertible lens to ensure the focal length free conversion within this range[2]. 


\section{Research on the calibration technology in mechanical vision detection technology}

See from the important index of mechanical vision detection technology system, calibration technology is an important part, it mainly means the camera calibration. When the mechanical vision detection technology system accesses to image information in the camera, calibration technology recognizes it through the specific location, size, geometrical information, shape of the computing object, and reflects the concrete strength of reflected light on calibration image. Considering that in the actual camera image collection process, the collect images may have distortion factor, therefore, camera calibration technology function here is to calculate the actual parameter of computer, then precisely correct distortion images according to the parameter, to finally ensure images can truly reflect camera object information. This is also the most important detection factor that can determine calibration accuracy in the visual machine detection technology system.

To be specific, set calibration process of Canon EOS 450D digital camera as example, list out its calibration algorithm implementation process, mainly divided into following 5 steps.

Step 1, first need to make black and white checkerboard plane template used in the calibration process, the specific method for making template is to use laser jet to print one piece of checkerboard sticker, and put it on the smooth plain glass, small checkerboard size of the template should be $20 \mathrm{~mm} * 20 \mathrm{~mm}$.

Step 2, fix the digital camera, adjust to the smallest the focal length on the calibration, and try to change the spacial location of the checkerboard plane template. At this time, choose 5 different places to take photos, final to get 5 calibration images that express different spacial location.

Step 3, on the basis of Harris sub-pixel level detection method to extract 24 grid vertexes from the calibration template images to match their 96 calibration feature points.

Step 4, use calibration calculation to calculate the actual calibration value of the digital camera, then get the distortion coefficient of camera lens from calibration value. The following table 1 shows the distortion coefficient and calibration result of camera lens.

\begin{tabular}{|c|c|}
\hline Distortion coefficient of the camera lens & Calibration result \\
\hline K1 & -0.172 \\
\hline K2 & 0.184 \\
\hline P1 & -0.004 \\
\hline P2 & 0.002 \\
\hline
\end{tabular}

Table 1 Distortion coefficient and calibration result of camera lens

Make external parameter setting for camera according to the calibration result, get all the 6 plane templates in the calibration process, and calibrate the corresponding location in the camera coordinates. Then use the calibration result to correct 6 calibration images correspondingly, get the corrected result.

Step 5, analysis on the calibration result error. Implement reprojection for all the 96 calibration points in the 6 plane calibration templates according to the calibration result of the camera. Among which, "+" in the calibration image represents the calibration location acquired by using Harris sub-pixel level detection method, "o" represents the specific location of calibration point after reprojection. Based on this to calibrate 576 reprojection points and observe to determine its reprojection error. Assume calibration point pixel coordinate is $\left(x_{i}, y_{i}\right)$, the reprojection pixel coordinate of calibration point is $\left(x_{i}^{\prime}, y_{i}^{\prime}\right)$, at this time, the average reprojection error of calibration point $(\Delta \bar{x}, \Delta \bar{y})$ can be calculated from the following formula:

$$
\Delta \bar{x}=\frac{\sum_{i=1}^{576}\left(x_{i}{ }^{\prime}-x_{i}\right)}{576}
$$




$$
\Delta \bar{y}=\frac{\sum_{i=1}^{576}\left(y_{i}^{\prime}-y_{i}\right)}{576}
$$

Based on above two formulas to calculate out the calibration reprojection error, final to calculate out the final calibration error through following formula:

$$
R M S=\sqrt{\frac{\sum_{i=1}^{576}\left(x_{i}^{\prime}-x_{i}\right)^{2}+\sum_{i=1}^{576}\left(y_{i}^{\prime}-y_{i}\right)^{2}}{576}}
$$

See from above elaboration that camera calibration precision truly will directly affect detection result precision, effective auxiliary for camera based on calibration calculation can get the final calibration value of the camera, which proves that the camera calibration has small error on result, improve calibration calculation to further improve calibration precision, final to meet demands of visual machine technology on the detection of steel plate surface defect[3].

\section{Research on the detection of steel plate surface defect based on the visual machine technology}

Steel plate surface defect mainly means scratch defect. This paper mainly explain the edge detection of the steel plate surface defect detection system based on visual machine technology and calculate the scratch length on the steel plate surface, final to analyze error.

\section{Research on the edge detection of steel plate surface scratch defect}

At present, normal machine vision steel plate surface defect detection system mainly uses the transform method of histogram balance and three level gray stretch, they can realize the strengthen treatment of detection images, then transform detection focus to the detection of steel plate scratch defect. Compare two methods, three level gray stretch transformation method is more accepted, because on the aspect of gray comparison, compared with the background, the steel plate scratch defect is more obvious and easier to be found, it is also more convenient for the effective extract of scratch defect.

Steel plate treated after this image treatment method, its surface image background with less noise, the scratch defect is more obvious, but still need to further calculate the scratch length on the steel plate and the total pixel on the length. Here better to use edge detection method to process surface image. After calculation, its scratch curve will be clear, which more embodied its real scratch length in the pixel picture[4].

\section{Real calculation process of the scratch length on the steel plate surface}

Generally speaking, image edge means the gray scale alteration in the local region of the image, its irregular changing area. We can treat the gray profile in this region as a phase step, its gray value will transform from the small changing area to that with big difference, final to form edge region concentrated in different gray value, it is also basic embody of image feature, can be treated as main feature for picture segmentation.

Calculation of the scratch length on the steel plate surface first needs to get through the refining process of image extracting to get main single pixel binary image of the steel plate surface scratch defect, and express its background as pixel 0 , while defect express as pixel 1. When calculate the actual physical length of scratch, calculate out all the point pixel distance A of the scratch length innovation covering pixel 1, surround center pixel as origin point to calculate pixel distance.At present, normally used pixel distance calculation method include euclidean distance calculation, adjacent distance calculation and so on, this paper use euclidean distance calculation.First based on plane calibration image to make precise calibration for camera lens, correctly express the real result and error of the reprojection of calibration point, then surround this calibration result to realize correction.Image needs to be cut after correction and extract the black check, make gray scale binary 
treatment, final to calculate the edge pixel distance $A^{t}$ trough euclidean distance calculation. Before calculation, we need to measure the physical size of the check edge (assume it is 10), then calculate the physical size B of single pixel on real space, get:

$$
B=\frac{10}{A}
$$

At last, calculate the real scratch length $L$ on the steel plate surface, its formula should be :

$$
L=A \times B=10 \times \frac{A}{A^{t}}
$$

\section{Error analysis}

According to the measured scratch length on steel plate surface to remeasure scratch by using edge segmentation, get the real scratch length $L^{t}$. At this time, use real measured scratch length and machine visual detection system to get the measurement error[5]:

$$
\delta=\frac{L^{\prime}-L}{L^{\prime}} \times 100 \%
$$

\section{Conclusion}

Automatic detection system of steel plate surface defect based on the machine visual technology shows its advantage on image collection, camera calibration technology and image processing, especially in the detection precision.It comprehensively considered the calibration mode perfection under the influence of image distortion, further improved the calibration precision of camera and improved calibration method to realize the high precision measurement og steel plate surface scratch defect.With the rapid development of machine visual technology, using this technology to realize automatic and highly effective detection has become a trend, which provided important support for the surface detection of steel plate raw material.This paper made simple analysis on key techniques in surface scratch detection from the technical perspective, future research process need to make a further analysis on the flaw, spot and other defects, realize dynamic detection on the steel plate surface defects, to realize the target of automatic control on detection result.

\section{References}

[1] He Yi. Application of Ultrasonic Sensor in the Baosteel Steel Coil Detection,. Electrotechnology,2003( 10) : 64-65.

[2] Guo Ping. Research on the Detection Technology for Steel Plate Surface Defect based on Machine Vision, Nanchang University, 2010.29-57.

[3] Han Fangfang. Research on the Online Detection Key Technology on Surface Defect Vision, Tianjin University, 2011.131-146.

[4] Cheng Wansheng. Research on the Detection Technology of Steel Plate Surface Defect, Harbin Institute of Technology,2008.122-126.

[5] Wu Xiaopeng, Lin Jiebang, Tang Hui etc., Research on the Detection Technology of Casting Blank Surface Defect based on Machine Vision, Wisco Technology, 2010,48(1):49-53. 